\title{
Dark matter or tidal disruption? The case for the Leo I dSph
}

\author{
S.T. Sohn ${ }^{1,2}$, S.R. Majewski ${ }^{2}$, J.C. Ostheimer ${ }^{2}$, W.E. Kunkel ${ }^{3}$, \\ R.J. Patterson ${ }^{2}$, M.H. Siegel ${ }^{2,4}$, P. Guhathakurta ${ }^{5}$ and M. Cooper ${ }^{6}$ \\ ${ }^{1}$ Korea Astronomy and Space Science Institute, Daejeon 305-348, Korea \\ email: tonysohn@kasi.re.kr \\ ${ }^{2}$ Department of Astronomy, University of Virginia, Charlottesville, VA 22903 \\ ${ }^{3}$ Las Campanas Observatory, Casilla 601, La Serena, Chile \\ ${ }^{4}$ McDonald Observatory, University of Texas, Austin, TX 78712 \\ ${ }^{5} \mathrm{UCO} /$ Lick Observatory, University of California at Santa Cruz, Santa Cruz, CA 95064 \\ ${ }^{6}$ Department of Astronomy, University of California at Berkeley, Berkeley, CA 94720
}

\begin{abstract}
We present results from photometric and spectroscopic survey of giant stars in the Leo I dwarf spheroidal galaxy. We find compelling evidence that this galaxy has been tidally disrupted.
\end{abstract}

Keywords. Galaxy: halo, galaxies: dwarf, galaxies: kinematics and dynamics, galaxies: Local Group

\section{Introduction}

The high apparent mass-to-light ratios $(M / L \mathrm{~s})$ of Local Group dwarf spheroidal galaxies (dSphs) raises an apparent dilemma in the context of a tidal disruption picture. If $\mathrm{dSph}$ galaxies are the visible counterparts of the largest dark matter (DM) lumps (Klypin et al. 1999), and the large measured $M / L$ of many dSphs truly reflect large internal DM contents, then the process of tidal disruption should be inhibited. The anticorrelation of dSph's intrinsic brightness with apparent $M / L$ has raised the suggestion that the mass of all dSphs are about the same (Mateo et al. 1998). From this standpoint, no dSph satellite might be expected to be tidally disrupted. The example of the Sgr dSph (e.g., Majewski et al. 2003) is clearly at odds with this, and that it may not be an exception is proposed by studies suggesting tidal disruption in other Mliky Way dSphs. Leo I, at the low end of the dSph $M / L$ scale $\left[6(M / L)_{\odot, V}\right.$; Mateo et al. 1998], provides an interesting contrasting case to the high $M / L$ systems in this DM/tidal disruption debate.

\section{Results}

We have surveyed a 4.5 degree $^{2}$ region centered on Leo I in $M, T 2,+D D O 51$ filter system to explore the extended morphology of this dSph. These photometric data were used to select Leo I giant candidates using the $(M-T 2, M-D D O 51)$ color-color and $(M-T 2, M)$ color-magnitude diagrams (see e.g. Majewski et al. 2000 for details). Using Leo I giant candidates, we explore two-dimensional distribution and find compelling evidence that Leo I has been tidally disrupted: (1) we find many Leo I giant candidates outside the King limiting radius, spread out predominantly to the east-west direction (Figure 1-a), (2) our isodensity contour plots show isophotal twists and hint of S-shaped morphology (Figure 1-b), and (3) we find double breaks in the radial density profile (Figure 1-c). We have also measured radial velocities (RVs) of 125 stars using spectra 

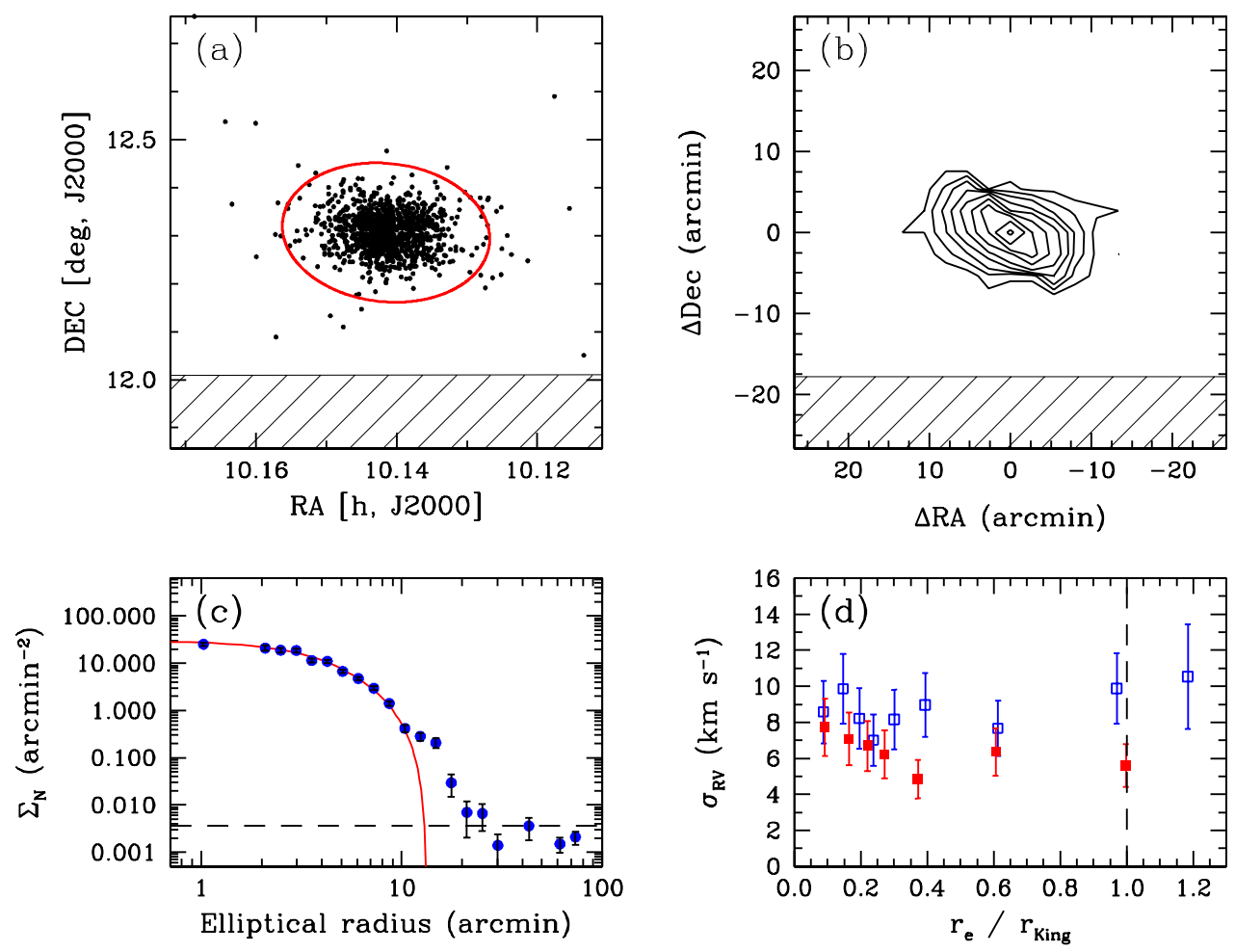

Figure 1. (a) A central region blowup of the distribution on the sky of the Leo I giant candidates. The ellipse shows the King limiting radius. The hatched region is outside of our survey area. (b) Isodensity contour plot of the stars in (a). (c) Fits of the stellar distribution of Leo I giant candidates using a King model. The dased line is the derived level of background, which was subtracted from the data and the model curve shown. (d) Variation of velocity dispersions with elliptical radius from the center of Leo I. The open squares are for the entire sample, while the closed squares are for the sample without the $2 \sigma$ outliers.

taken with the Keck II + DEIMOS. Out of 105 Leo I members, 28 stars lie at $1.0<$ $r_{e} / r_{\text {King }}<1.7$, confirming the existence of the break population found in the radial surface density profile. The RV dispersion profile (Figure 1-d) for the more Gaussiandistributed RV population decrease with distance from the center out to $r_{e} / r_{\text {King }} \sim 0.4$, and flattens beyond that. The profile for the entire sample, however, is flat (or slightly rising) out to the King limiting radius and beyond.

Based on the results found in both photometric and spectrocopic surveys, we conclude that we are seeing a tidal disruption in the Leo I system.

\section{References}

Klypin, A., Kravstov, A.V., Valenzuela, O. \& Prada, F. 1999, ApJ 522, 82

Majewski, S.R., Ostheimer, J.C., Patterson, R.J., Kunkel, W.E., Johnston, K.V. \& Geisler, D. 2000, $A J 119,760$

Majewski, S.R., Skrutskie, M.F., Weinberg, M.D. \& Ostheimer, J.C. 2003, ApJ 599, 1082

Mateo, M., Olszewski, E.W., Vogt, S.S. \& Keane, M.J. 1998, AJ 116, 2315 